\title{
Photoelectric effect with a twist
}

Giovanni De Ninno ${ }^{1,2}$, Jonas Wätzel ${ }^{3}$, Primož Rebernik Ribič ${ }^{1,2}$, Enrico Allaria ${ }^{1}$, Marcello Coreno ${ }^{4,1}$, Miltcho B. Danailov ${ }^{1}$, Christian David ${ }^{5}$, Alexander Demidovich ${ }^{1}$, Michele Di Fraia ${ }^{1}$, Luca Giannessi ${ }^{1,6}$, Klavs Hansen ${ }^{7,8}$, Špela Krušič ${ }^{9}$, Michele Manfredda ${ }^{1}$, Michael Meyer ${ }^{10}$, Andrej Mihelič ${ }^{9}$, Najmeh Mirian ${ }^{1}$, Oksana Plekan ${ }^{1}$, Barbara Ressel $^{2}$, Benedikt Rösner ${ }^{5}$, Alberto Simoncig ${ }^{1}$, Simone Spampinati ${ }^{1}$, Matija Stupar $^{2}$, Matjaž Žitnik ${ }^{9}$, Marco Zangrando ${ }^{1,11}$, Carlo Callegari ${ }^{1}$, Jamal Berakdar ${ }^{3}$

${ }^{1}$ Elettra-Sincrotrone Trieste S.C.p.A., 34149 Trieste, Italy

${ }^{2}$ University of Nova Gorica, 5000 Nova Gorica, Slovenia

${ }^{3}$ Institut für Physik, Martin-Luther Universität Halle-Wittenberg, 06099 Halle (Saale), Germany

${ }^{4}$ ISM-CNR, in Basovizza Area Science Park, 34149 Trieste, Italy

${ }^{5}$ Paul Scherrer Institut, 5232 Villigen-PSI, Switzerland

${ }^{6}$ INFN-LNF, Via E. Fermi 40, 00044 Frascati (Rome), Italy

${ }^{7}$ Center for Joint Quantum Studies and Department of Physics, Tianjin University, 92 Weijin Road, Tianjin 300072, China

${ }^{8}$ Dept. of Physics, Gothenburg University, 41296 Gothenburg, Sweden

${ }^{9} \mathrm{~J}$. Stefan Institute, Jamova cesta 39, SI-1000 Ljubljana, Slovenia

${ }^{10}$ European XFEL GmbH Holzkoppel 422869 Schenefeld, Germany

${ }^{11}$ Istituto Officina dei Materiali, Consiglio Nazionale delle Ricerche, Basovizza, Italy.

Photons have fixed spin and unbounded orbital angular momentum (OAM). While the former is manifested in the polarization of light, the latter corresponds to the spatial phase distribution of its wave front [1]. The distinctive way in which the photon spin dictates the electron motion upon light-matter interaction is the basis for numerous well-established spectroscopies that reveal the electronic, magnetic and structural properties of matter. In contrast, imprinting OAM on a matter wave, specifically on a propagating electron, is generally considered very challenging and the anticipated effect undetectable [2]. Indeed, this amounts to transferring the phase of a classical electromagnetic wave, defined within several hundreds of nanometres, to a quantum particle localized within the few angstroms of an atom. In addition, the centre of symmetry of irradiated atoms does not in general coincide with the axis of the photon beam. In [3], the authors provided evidence of OAM-dependent absorption of light by a cold trapped atom, located in the centre of the light beam. Off-centre excitation was studied in [4]. Here we seek to observe an OAM-dependent dichroic photoelectric effect, using an extended sample of He atoms. Surprisingly, we find experimentally, and confirm theoretically, that the OAM of an optical field can be imprinted coherently onto a propagating electron wave, and that this phase information survives ensemble averaging out to macroscopic distances, where the electron is detected. We also show that electronic transitions, which are otherwise optically inaccessible due to selection rules, are essential for this process to occur. Our results reveal new aspects of light-matter interaction and point to a new kind of single-photon electron spectroscopy for accessing electronic optical transitions that are usually forbidden by symmetry.

In our experiment, He atoms are ionized by XUV radiation, generated by a freeelectron laser (FEL) [5], in the presence of an intense infrared (IR) laser field, see Fig. 
1a. The FEL beam is in a fixed (right-handed) circular polarization state, $\sigma_{\mathrm{FEL}}=+1$. What happens if the IR field has a vortex phase profile carrying OAM? Is it possible to observe a vortex-dependent dichroic effect that depends on the sign of the OAM, even though the atoms are randomly distributed across the profile of the OAM beam? This would be surprising: the atomic wave function is extremely localized on the scale of the OAM beam's waist, see Fig. 1b. Off-axis atoms experience the IR laser field as an ordinary Gaussian beam [6] and atoms close to the optical axis, where the OAM is well defined, experience a vanishingly small field [7]; besides, the fraction of near-axis atoms is small. While the transfer of optical OAM to photoelectrons seems unlikely, if it happens, it should involve a new type of non-dipole transitions [7,6], related to the OAM-carrying vector potential, which could be used, e.g., to optically induce orbital magnetization in molecules [8].

To investigate the possibility of observing a vortex-dependent dichroic effect on randomly distributed electrons, we used an IR laser with adjustable right or lefthanded circular polarization $\left(\sigma_{\mathrm{IR}}= \pm 1\right)$, and variable amount $m_{\mathrm{OAM}} \hbar$ of OAM, where $m_{\mathrm{OAM}}$ is a signed integer, called topological charge. The two light beams are focused and overlapped with a gas jet of He atoms in a vacuum chamber equipped with a velocity map imaging (VMI) spectrometer [9]. The velocity distribution of electrons produced in the interaction volume is projected onto a 2D imaging detector. For high enough IR intensities, the resulting photoelectron spectra, see Figs. 1c and 1d, show a main band, corresponding to direct (XUV-induced) photoemission, together with a series of weaker rings, called sidebands, whose radius increment (decrement) represents the energy acquired (lost) by the photo-emitted electrons upon absorption (emission) of one or more IR photons. All bands depend on the polar angle at which photoelectrons are emitted $[10,11]$. They reflect the interplay between XUV and optical fields during the photoionization process [12,13], providing information on the transfer of light properties, e.g., angular momentum, to matter [14-17]. When the two beams are circularly polarized and $m_{\mathrm{OAM}}=0$, there is a few-percent difference in the angular electron distributions for co-rotating and counter-rotating XUV and IR fields, resulting in a dichroism contrast in the range 5-10\% [18-21].

Now what happens if $m_{\mathrm{OAM}}= \pm 1$. The two VMI spectra shown in Figs. 1c and $1 \mathrm{~d}$ have been obtained for fixed IR polarization $\left(\sigma_{\mathrm{IR}}=+1\right)$ and alternate OAM $(+\hbar$ and $-\hbar$, respectively), with all other experimental conditions kept unchanged. The images demonstrate that different topological charges result in qualitatively different photoelectron spectra, attesting a clear OAM-dependent dichroic effect. This is a first proof of the possibility to imprint virtually unbounded photon OAM on the matter wave of a propagating electron.

To clarify the underlying physics of OAM-dependent dichroism we developed a firstprinciple theoretical model that accounts for all non-linear effects in light-matter interaction (see Supplementary Material) and amounts to a numerical solution of the three-dimensional, time-dependent Schrödinger equation for the lasers-driven atom, and an averaging over the atoms ensemble in the lasers spots. Analytically, insight into the important contributions is possible by considering a single atom centred on the optical axis and a weak IR field. Ionization upon absorption of one XUV photon with frequency $\omega_{\text {FEL }}$ followed by the absorption of one IR photon with frequency $\omega_{\text {IR }}$ is described by the two-photon ionization amplitude:

$$
M\left(f, \omega_{F E L}, \omega_{I R}\right)=\frac{1}{i} \lim _{\varepsilon \rightarrow 0^{+}} £_{v} \frac{\left\langle f\left|H_{\mathrm{int}}^{\mathrm{IR}}\right| v\right\rangle\left\langle v\left|H_{\mathrm{int}}^{\mathrm{FEL}}\right| 1 \mathrm{~s}\right\rangle}{E_{1 \mathrm{~s}}+\omega_{\mathrm{FEL}}-E_{v}+i \varepsilon} .
$$


The electron is excited from the ground state $|1 \mathrm{~s}\rangle$, having energy $E_{1 \mathrm{~s}}$, to the final state $|f\rangle$, passing through intermediate states $|v\rangle$, having energies $E_{v}$. Both lasers propagate in the same direction, which is taken as the quantization axis. The dipole transition due to the FEL-atom interaction, mediated by $H_{\mathrm{int}}^{\mathrm{FEL}}$, determines the angular distribution of the (continuum) intermediate states $|v\rangle$, described by the spherical harmonics $Y_{l=1, m=\sigma_{\mathrm{FEL}}}\left(\vartheta_{k}, \varphi_{k}\right)$, where $\left(\vartheta_{k}, \varphi_{k}\right)$ are the polar and azimuthal spherical angles specifying the electron linear-momentum $(\boldsymbol{k})$ direction. The quantum numbers $l=1, m=\sigma_{\mathrm{FEL}}$ reflect the symmetry of the electric dipole operator. The corresponding photoelectron distributions are shown in the middle part of Figs. 2a and $2 \mathrm{~b}$. The OAM is transferred to the electron via a continuum-continuum transition through the action of the IR-atom interaction matrix element, $\left\langle f\left|H_{\mathrm{int}}^{\mathrm{IR}}\right| v\right\rangle$. Hence, the energy and angular distribution of the final state is encoded into the first outer (IR absorption) or inner (IR emission) sideband. It can be shown (see Methods) that at short distance, $\rho$, from the optical axis, $H_{\text {int }}^{\mathrm{IR}}$ can be decomposed into a contribution stemming from the longitudinal electric field component [22], scaling as $(\rho /$ $\left.w_{0}\right)^{\left|m_{\mathrm{OAM}}+\sigma_{\mathrm{IR}}\right|}$, where $w_{0}$ is the waist of the IR beam, and another due to the transverse field component, scaling as $\left(\rho / w_{0}\right)^{\left|m_{\mathrm{OAM}}\right|}$. The interplay between photon $\mathrm{OAM}$ and spin plays a crucial role: in the parallel case, i.e., for $m_{\mathrm{OAM}}=\sigma_{\mathrm{IR}}=+1$, the longitudinal component scales as $\left(\rho / w_{0}\right)^{2}$, and the transverse component like $\left(\rho / w_{0}\right)$. Near the optical axis, where $\left(\rho / w_{0}\right) \ll 1$, the continuum-continuum matrix element $\left\langle f\left|H_{\text {int }}^{\mathrm{IR}}\right| v\right\rangle$ contributes with a dominant quadrupole transition, governed by the selection rule $\Delta l=2, \Delta m=2$, leading to an asymptotic momentum distribution proportional to $Y_{3,3}\left(\vartheta_{\boldsymbol{k}}, \varphi_{\boldsymbol{k}}\right)$. As shown in the top part of Fig. 2a, such a function has a donut-like shape, extending in the transverse plane. In contrast, in the antiparallel case, i.e., for $\sigma_{\mathrm{IR}}=-m_{\mathrm{OAM}}=+1$, a strong on-axis longitudinal component scaling as $\left(\rho / w_{0}\right)^{0}=1$ is manifested, while the transverse component scales again as $\left(\rho / w_{0}\right)$. Consequently, the latter can be neglected and the continuum-continuum transition is dominated by a dipolar term, with the selection rule $\Delta l=1, \Delta m=0$, which forms the electron density in the longitudinal direction, see top part of Fig $2 b$. The different combinations of $\sigma_{\mathrm{IR}}$ and $m_{\mathrm{OAM}}$ we have considered in this work, together with the selection rules for the corresponding transitions, are shown in the inset of Fig. $2 b$.

The projection of the solution of the Schrödinger equation on above-threshold continuum states yields the differential cross sections (DCSs) for a given electron kinetic energy. Therefore, the calculated DCSs can be directly compared to the sideband area, as a function of the polar angle, obtained experimentally using the VMI spectrometer. However, in order to make this comparison possible, one needs to move from the single-atom picture to the real-world ensemble average. For that purpose, the problem is solved for atoms randomly distributed in the whole FEL and IR spots and the results are then averaged. As shown in Figs. 3a and 3b, the average does not substantially change the shape of the electron distribution when the spin and OAM are parallel, while it has a quite evident effect when the momenta are antiparallel. In the latter case, as shown in Fig. 3c, the calculated average DCS for the first outer sideband shows a peak in the transverse direction (i.e., $\vartheta_{\boldsymbol{k}}=\pi / 2$ ), whereas the single-electron DCS (Fig. 2b, top part) shows a minimum. However, as shown in Fig. 3d, a clear dichroic effect is still visible. The insets of Figs. 3a and $3 \mathrm{~b}$ show the projection of the photoelectron distributions on the $\left(k_{y}, k_{z}\right)$ plane. Such projections can be directly compared to the experimental VMI images. 
For a more direct comparison with theoretical results, we collected data at low IR intensity, enabling a straightforward interpretation of the VMI images by means of first-order processes. The VMI image obtained for the case $\sigma_{\mathrm{IR}}=+1, m_{\mathrm{OAM}}=-1$ is shown in Fig. 4a, while Fig. 4b shows the corresponding theoretical prediction. The qualitative agreement is very good. By acquiring data for different combinations of the IR spin and OAM, we extracted the DCS for the first sideband, after Abel inversion of the VMI spectra [23]. As a benchmark, we first considered the case $\sigma_{\mathrm{IR}}= \pm 1$, with $m_{\mathrm{OAM}}=0$ (i.e., standard, spin-dependent, dichroism). We obtained a good agreement between experiments and theory, and results similar to the ones reported in [21] (data not shown). Then, we focused on the cases wit $h$ nonzero OAM. Fig. $4 \mathrm{c}$ shows the DCSs for the cases $\sigma_{\mathrm{IR}}=+1, m_{\mathrm{OAM}}=+1$ and $\sigma_{\mathrm{IR}}=$ $+1, m_{\mathrm{OAM}}=-1$, together with the OAM-dependent dichroism as a function of the polar angle. Fig. $4 \mathrm{~d}$ reports the corresponding theoretical predictions. The agreement between experiment and theory is good: in both cases there is a clear OAM-dependent dichroic effect, which is maximum, i.e., about $(7.5 \pm 1.2) \%$, at about $\vartheta_{k}=\pi / 2$ (for a discussion on the evaluation of both statistical and systematic errors, see Methods). A similar good agreement was found for switched OAM and switched polarizations, see Figs. 4e and 4f, and for fixed OAM and switched polarizations, see Figs. 4g and $4 \mathrm{~h}$. Note that in the former case nonzero dichroism is present at all angles, in the range 3$7 \%$, depending on the value of $\vartheta_{k}$, while in the latter significant dichroism, about $(2.5 \pm 1.3) \%$, is observed at $\vartheta_{k}=\pi / 4$ and $\vartheta_{k}=3 \pi / 4$.

Summarizing, we confirm that the spatial distribution of an optical field with vortex phase profile can be imprinted coherently on a photoelectron wave packet that recedes from atoms randomly distributed over the laser spot. The large dimension acquired by the electron wave function during the two-colour continuum-continuum process is the key for explaining the success of our experiment, in spite of the very different size of atoms and OAM beam's waist. Indeed, the OAM-carrying IR beam acts on the electron wave function after the latter is inflated, following the XUV-induced atom ionization. The propagating photoelectron wave can scan over the spatial topology of the IR field, acquiring OAM. Instead, a direct ionization with XUV OAM photons produces essentially OAM-independent photoelectrons [2]. The observed effect is traced back to non-dipole transitions and to the transverse component of the light wave. Larger dichroic contrasts may be expected by using a probe beam carrying higher OAM, or by considering higher-order sidebands, corresponding to multiphoton absorption. Our results explore new aspects of light-matter interaction and point to qualitatively novel analytical tools, which can be used (e.g.) to study the instric chiral structure of compex organic molecules.

\section{Methods}

Experiment. The experiment was carried out at the LDM beam line of the FERMI FEL [5]. Right-hand circularly polarized XUV pulses at $28.5 \mathrm{eV}(43.5 \mathrm{~nm})$, with a bandwidth of about $94 \mathrm{meV}(0.05 \mathrm{~nm})$ and a duration of about $100 \mathrm{fs}$ were delivered by APPLE II-type undulators, via the Photon Analysis Delivery and REduction System (PADRES) [24]. The average pulse energy was $80 \pm 10 \mu \mathrm{J}$, with a repetition rate of $10 \mathrm{~Hz}$. Active mirrors in Kirkpatrick-Baez configuration [25] focused the FEL pulses to a spot size of about $10 \mu \mathrm{m}$ diameter (full width at half maximum). Taking into account the theoretical overall transmission of all optical components in the beamline (about 60\%), average intensities up to $10^{18} \mathrm{~W} / \mathrm{m}^{2}$ were reached in the interaction region [26]. Near IR pulses from a Ti:Sa laser $(1.58 \mathrm{eV} ; 784 \mathrm{~nm})$ were 
used, with variable (right and left-handed, $\sigma_{\mathrm{IR}}= \pm 1$ ) circular polarization, generated using a combination of a half wave plate and a quarter wave plate, and variable topological charge $\left(m_{\mathrm{OAM}}= \pm 1\right)$, generated using a phase mask. The IR pulse bandwidth was $13 \mathrm{~nm}$ (full width at half maximum) and its duration $175 \mathrm{fs}$. The pulse energy was varied in the range $120-800 \mu \mathrm{J}$. With a spot size of $60 \pm 10 \mu \mathrm{m}$, and taking into account optics efficiency and transmission, the intensity range at the sample position was $0.5-4 \times 10^{17} \mathrm{~W} / \mathrm{m}^{2}$. The highest IR pulse energy was used for the initial optimization and to obtain a first evidence of OAM-dependent dichroism (see Figs. 1c and 1d). Subsequently, a lower intensity (about $0.7 \times 10^{17} \mathrm{~W} / \mathrm{m}^{2}$ ) was employed for collecting the results to be compared with theoretical predictions. The IR pulses were produced by splitting the FEL-seeding pulses, providing a very stable two-colour synchronization. The FEL and the IR pulses were overlapped in time and space in the centre of the experimental chamber. The spatial overlap was determined by visual inspection of the images of both beams on a retractable Ce:YAG fluorescent screen. The system is based on a commercial digital camera and two achromatic doublets in a finite/finite-conjugate symmetric arrangement. The resolution of the optical system is $7 \mathrm{um}$, and a conservative estimate of the accuracy of visual overlap is $20 \mu \mathrm{m}$. The spatial overlap was also checked periodically by seeking the local minimum of the sideband signal while steering the IR beam. As for the case of visual overlap, a conservative estimate of the accuracy of this procedure is $20 \mathrm{um}$. The temporal overlap was controlled by a delay stage in the IR-laser delivery system and was optimized using first a coaxial cable antenna and then directly the two-colour twophoton signal. The VMI spectrometer includes a 75-mm diameter imaging quality multichannel plate (MCP) stack + phosphor screen, with a sCMOS camera for the image readout. Its electron optics were set to work optimally for the selected kinetic energies of $\mathrm{He} 1 \mathrm{~s}$ electrons. A pulsed, supersonic $\mathrm{He}$ beam produced with a commercial Even-Lavie valve (aperture $260 \mu \mathrm{m}$ ) was used to provide sufficient gas density (about $10^{16}$ atoms per $\mathrm{m}^{3}$ ) in the interaction region, allowing a shot-to-shot analysis of the VMI spectra.

Data analysis. The sCMOS camera $(2,560 \times 2,160$ pixels; $6.5 \mu \mathrm{m}$ pixel size $)$ readout acquired single-shot pictures of the two-dimensional projection of the electron angular distribution [9]; only the central portion $(900 \times 900$ pixels, corresponding to the maximum data flow that the camera can handle at the full repetition rate of the FEL) was acquired, corresponding to the full size of the phosphor screen. For low IR energy, i.e., for strong suppression of the signal of sidebands higher than one (see for example, Fig. 4a), these images were transformed into three dimensional momentum distributions with full angular reconstruction of the electron emission, by applying an Abel transformation [23]. The transformed images were then analysed by means of a MATLAB script, allowing to calculate the photoelectron yield of the first sideband, as a function of the polar angle. The analysis focused on the first outer sideband; the inner sideband, though carrying the same information, is affected by the overlap with the signal from the main band in the two-dimensional VMI image, leading to a higher statistical error. Dichroism was calculated as the difference between the DCSs corresponding to two specific sets of photon spin and OAM, normalized to the sum of the maxima of the same DCSs. The statistical error on the dichroic contrast (see Fig. 4) was estimated by calculating the number of electrons at some representative polar angles (i.e., $\vartheta_{k}=\pi / 2, \pi / 4,3 / 2 \pi$ ) of the ring corresponding to the first sideband of the VMI images and assuming a Poisson-like error distribution. The number of electrons 
located at $\vartheta_{k}=\pi / 2$ is typically of the order of $3.5 \times 10^{3}$, giving an error of about $1.7 \%$; the number of electrons located at $\vartheta_{k}=\pi / 4$ and $\vartheta_{k}=3 / 2 \pi$ is of the order of $10^{3}$, giving an error of about $3.2 \%$. The uncertainty on the dichroic contrast, at a given $\vartheta_{k}$, was calculated by error propagation. The profile of the dichroic contrast is also affected by a systematic error due to the lack of perfect reproducibility in the procedure for the transverse alignment between the XUV and IR beams, which was carried out each time, after flipping the OAM phase mask. As it was already mentioned, this procedure has an estimated accuracy of $20 \mu \mathrm{m}$. Such an error is likely to be at the origin of the small distortion in the profile of the dichroic contrasts shown in Fig. 4.

Theoretical model. Within the single-active-electron approach, the time-dependent three-dimensional Schrödinger equation was solved numerically on a fine space-time grid ( $\Delta r=0.05$ a.u. and $\Delta t=0.025$ a.u.), using an effective potential [27] and in the presence of both laser fields. Our method relies on the representation of the wave function in a spherical basis, i.e., using spherical coordinates $\boldsymbol{r}=\{r, \vartheta, \varphi\}$ relative to atom's core: $\Psi(\boldsymbol{r}, t, \boldsymbol{b})=\sum_{l, m} R_{l, m, \boldsymbol{b}}(r, t) Y_{l, m}(\vartheta, \varphi)$. Here, $l$ and $m$ are the orbital and magnetic quantum numbers, respectively, while $R_{l, m}(r)$ is the radial wave function for the angular channel $l, m$ and $Y_{l, m}(\vartheta, \varphi)$ are spherical harmonics. The parameter $\boldsymbol{b}$ indicates the position of the considered atom relative to the (common) optical axes of both laser fields. The initial condition is given by $\Psi(r, t \rightarrow-\infty, \boldsymbol{b})=$ $\mathcal{R}_{1 \mathrm{~s}, \boldsymbol{b}}(r) Y_{0,0}(\vartheta, \varphi)$, where $\mathcal{R}_{1 \mathrm{~s}, \boldsymbol{b}}(r)$ is the radial wave function of the 1 s electron in the He ground state, at the shifted position $\boldsymbol{b}$. The wave-function is propagated to a time $t=T_{\mathrm{obs}}$, at which both laser fields are extinguished. Then, $\Psi(\boldsymbol{r}, t, \boldsymbol{b})$ is projected (7) onto a set of continuum states $\left|\phi_{\boldsymbol{k}}^{(-)}\right\rangle$, characterized by the asymptotic momentum $\boldsymbol{k}$ (with direction $\left\{\vartheta_{k}, \varphi_{k}\right\}$ ), to obtain the DCS, i.e. $\frac{d \sigma_{b}}{d \Omega_{k}}=$ $\left|\left\langle\phi_{\boldsymbol{k}}^{(-)} \mid \Psi\left(T_{\mathrm{obs}}, \boldsymbol{b}\right)\right\rangle\right|^{2}$, where $d \Omega_{\boldsymbol{k}}=\sin \vartheta_{\boldsymbol{k}} d \vartheta_{\boldsymbol{k}} d \varphi_{\boldsymbol{k}}$

273 Crucial is a proper description of the complete light-matter interaction Hamiltonian 274 operator which consists of two contributions: (i) $\widehat{H}_{\mathrm{int}}^{\mathrm{FEL}}(t)=\boldsymbol{A}_{\mathrm{FEL}}(\boldsymbol{r}-\boldsymbol{b}, t) \cdot \boldsymbol{p}+$ $275 \frac{1}{2} \boldsymbol{A}_{\mathrm{FEL}}(\boldsymbol{r}-\boldsymbol{b}, t)^{2}$ describing the action of the Hermite-Gaussian FEL pulse and (ii) $\widehat{H}_{\mathrm{int}}^{\mathrm{IR}}(t)=\frac{1}{2}\left[\boldsymbol{p} \cdot \boldsymbol{A}_{\mathrm{IR}}(\boldsymbol{r}-\boldsymbol{b}, t)+\boldsymbol{A}_{\mathrm{IR}}(\boldsymbol{r}-\boldsymbol{b}, t) \cdot \boldsymbol{p}+\boldsymbol{A}_{\mathrm{IR}}(\boldsymbol{r}-\boldsymbol{b}, t)^{2}\right]-\Phi_{\mathrm{IR}}(\boldsymbol{r}-\boldsymbol{b}, t)$ for the vortex IR field. Here, we introduced the momentum operator $\boldsymbol{p}$, while $\boldsymbol{A}_{\mathrm{FEL}}(\boldsymbol{r}, t), \boldsymbol{A}_{I R}(\boldsymbol{r}, t)$ and $\Phi_{I R}(\boldsymbol{r}, t)$ are the electromagnetic vector and scalar potentials of the FEL and IR fields. The final DCS, which is compared to the experimental VMI images, is obtained after averaging over $N_{A}$ randomly distributed atoms within the FEL spot, i.e., $\mathrm{DCS}_{\text {total }}=\frac{1}{N_{A}} \sum_{\boldsymbol{b}} \frac{d \sigma_{\boldsymbol{b}}}{d \Omega_{\boldsymbol{k}}}$.

282 The vector potential of the IR vortex field with photon energy $\hbar \omega_{\mathrm{IR}}$ (wave number $\left.283 k_{\mathrm{IR}}=\omega_{\mathrm{IR}} / c\right)$ is given by $\boldsymbol{A}_{\mathrm{IR}}(\boldsymbol{r}, t)=A_{0}^{\mathrm{IR}} \boldsymbol{\epsilon}_{\mathrm{IR}} u(\boldsymbol{r}) f(t) e^{i\left(k_{\mathrm{IR}} z-\omega_{\mathrm{IR}} t\right)}+$ c. c., where $A_{0}^{\mathrm{IR}}$ is the amplitude and the transversal distribution function reads

$$
u(\boldsymbol{r})=\left(\frac{\sqrt{2} \rho}{\widetilde{w}(z)}\right)^{m_{\mathrm{OAM}}} e^{-\frac{\rho^{2}}{\widetilde{w}^{2}(z)}} e^{i\left[m_{\mathrm{OAM}} \varphi+\frac{k_{\mathrm{IR}} \rho^{2} z}{2\left(z^{2}+z_{R}^{2}\right)}-\left(m_{\mathrm{OAM}}+1\right) \tan ^{-1} z / z_{R}\right]},
$$

with $\rho=r \sin \vartheta$ and $z=r \cos \vartheta$. Further, we introduce the beam waist $w_{\mathrm{IR}}$ which determines both the beam width $\widetilde{w}(z)=w_{\mathrm{IR}} \sqrt{1+z^{2} / \tilde{z}_{R}^{2}}$, as well as the Rayleigh range $\tilde{z}_{R}=k_{\mathrm{IR}} w_{\mathrm{IR}}^{2} / 2$. The vector field is not solenoidal. Depending on the circular polarization state (governed by $\boldsymbol{\epsilon}_{\mathrm{IR}}=-\sigma_{\mathrm{IR}}\left[\boldsymbol{\epsilon}_{x}+i \sigma_{\mathrm{IR}} \boldsymbol{\epsilon}_{y}\right] / \sqrt{2}$, where $\boldsymbol{\epsilon}_{x}$ and $\boldsymbol{\epsilon}_{y}$ are the 
Cartesian unit vectors) relative to the carried OAM, the emitted photoelectron may be exposed to a strong scalar potential $\Phi_{\mathrm{IR}}(\boldsymbol{r}, t)$ (action of a longitudinal field). The leading term scales as $\Phi_{\mathrm{IR}}(\boldsymbol{r}, t) \sim \frac{c}{k_{\mathrm{IR}}}\left(\frac{\rho}{w_{\mathrm{IR}}}\right)^{m_{\mathrm{OAM}}+\sigma_{\mathrm{IR}}} e^{i k_{\mathrm{IR}} z}$ while the (transverse) vector potential scales as $\mathrm{A}_{\mathrm{IR}}(\boldsymbol{r}, t) \sim\left(\frac{\rho}{w_{\mathrm{IR}}}\right)^{m_{\mathrm{OAM}}}$ in the vicinity of the optical axis. As a consequence, in the parallel case, i.e., for $m_{\mathrm{OAM}}=\sigma_{\mathrm{IR}}$, the transverse contribution of the interaction Hamiltonian $\widehat{H}_{\mathrm{int}}^{\mathrm{IR}}(t)$ dominates the continuum-continuum transition to the side band energy. Indeed, under the present experimental conditions, $(\rho /$ $\left.w_{\mathrm{IR}}\right) \ll 1$ ( $w_{\mathrm{IR}}$ being in the $\mu \mathrm{m}$ regime). Conversely, in the antiparallel case, i.e., for $m_{\mathrm{OAM}}=-\sigma_{\mathrm{IR}}$, the electric scalar potential dominates and leads to a dipole transition. Writing the interaction Hamiltonian in terms of spherical harmonics, the nature of the electronic transitions to the first side band, in the presence of the IR laser and for $\boldsymbol{b}=$ 0 , becomes evident. The dominating first-order transitions, see inset of Fig. $2 b$, are governed by the following selection rules, depending on the OAM and spin states of the IR photon:

(i) $\quad m_{O A M}=\sigma_{I R}=+1:|l=1, m=1\rangle \stackrel{\text { IR }}{\rightarrow}|l=3, m=3\rangle$, quadrupole transition induced by the transverse component of the interaction Hamiltonian; rule: $\Delta l=2, \Delta m=2$;

(ii) $\quad m_{\mathrm{OAM}}=-\sigma_{\mathrm{IR}}=+1:|l=1, m=1\rangle \stackrel{\mathrm{IR}}{\rightarrow}|l=2, m=1\rangle$, dipole $\quad$ transition induced by the electric scalar potential (longitudinal field); rule: $\Delta l=1$, $\Delta m=0$;

(iii) $\quad m_{O A M}=\sigma_{I R}=-1:|l=1, m=1\rangle \stackrel{\text { IR }}{\rightarrow} \alpha|l=1, m=-1\rangle+\beta|l=3, m=-1\rangle$

(where $\alpha$ and $\beta$ are complex coefficients), quadrupole transition induced by the transverse component of the interaction Hamiltonian; rule: $\Delta l=0,2$, $\Delta m=-2$.

Acknowledgments We acknowledge the support of the project "Triggering forbidden phenomena with twisted light and particle beams" (No J1-8134), funded by the Slovenian Research Agency (ARRS), and of the EU-H2020 project NFFA (grant agreement No 654360). Theoretical study has been financed by the German Science Foundation (DFG), within the priority program 1840 "Quantum dynamics in tailored intense fields".

Author contributions GDN, PRR, JW and JB proposed the experiment, whose feasibility was discussed with MC, MŽ and CC. GDN coordinated the experiment. JW and JB developed the theoretical model and JW carried out the simulations. CC, $\mathrm{AM}, \mathrm{BR}, \mathrm{JW}, \mathrm{KH}, \mathrm{MC}, \mathrm{MDF}, \mathrm{MS}, \mathrm{OP}, \breve{S} \mathrm{~K}, \mathrm{PRR}$ and GDN carried out the measurements. PRR, EA, AD, AS, BR, CD, MBD, MDF, MMa, MZ, NM and SS prepared the light source. GDN, BR and MDF carried out the data analysis. GDN, JW and JB wrote the first draft of the manuscript; the latter was first reviewed by AM, $\mathrm{BR}, \mathrm{CC}, \mathrm{MMe}, \mathrm{MŽ}$ and PRR and then discussed with all co-authors. 
Figures

346

347 


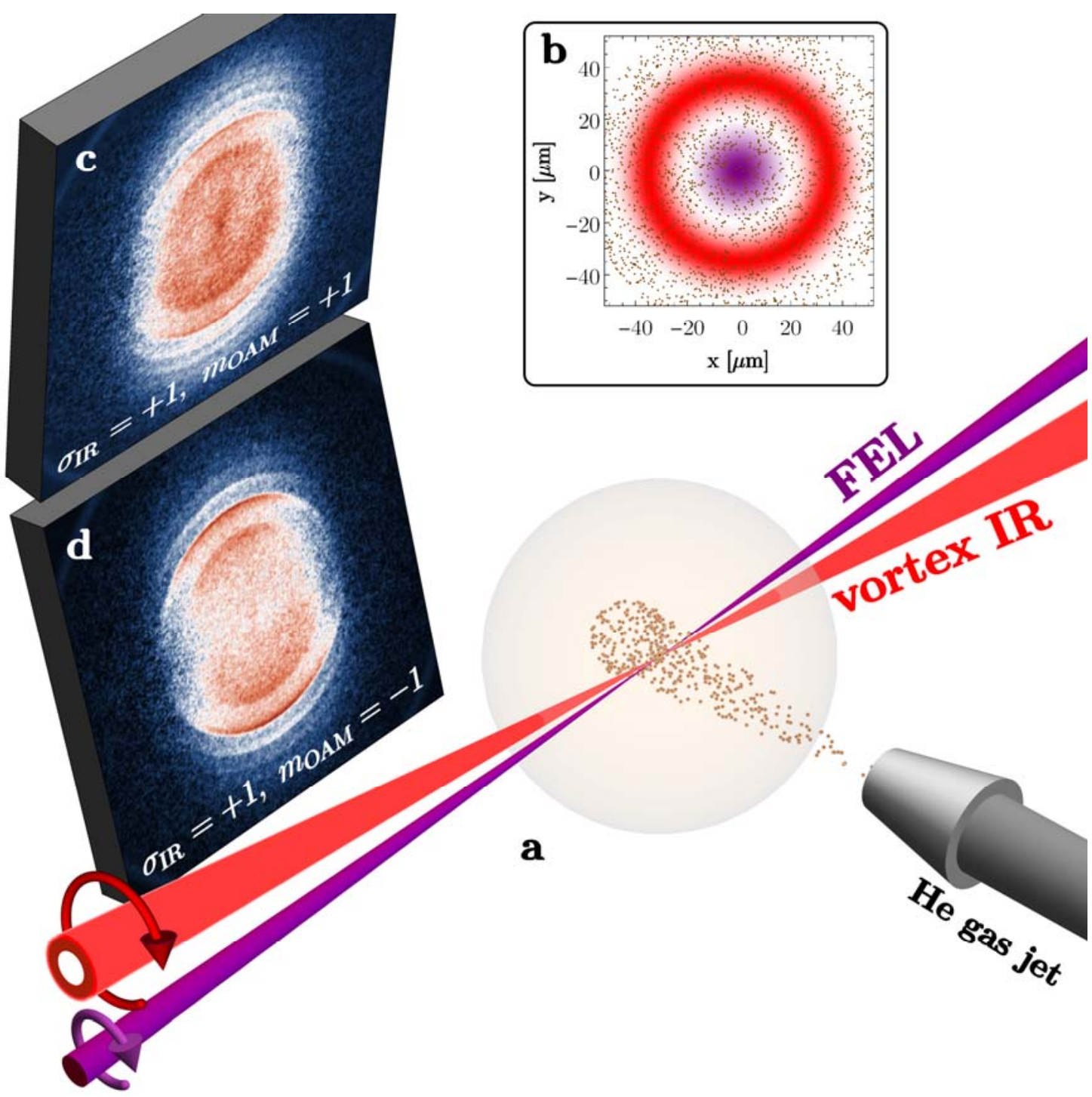

Fig. 1 | Experimental setup and first evidence of OAM-dependent dichroism. a, Schematic representation of the experimental setup. The XUV light pulse is generated using a FEL. It has fixed (right-handed) circular polarization, a spot size of about 10 $\mu \mathrm{m}$ and intensity (at the sample position) of the order of $10^{18} \mathrm{~W} / \mathrm{m}^{2}$. The infrared laser beam has a spot size of about $60 \mu \mathrm{m}$ and a power density in the range $0.5-4 \times 10^{17}$ $\mathrm{W} / \mathrm{m}^{2}$. It provides variable circular (right and left-handed) polarization and can be made to carry a topological charge $\left(m_{\mathrm{OAM}}= \pm 1\right)$. Helium gas is injected into the experimental chamber (at room temperature) via a gas jet system. A VMI spectrometer is used to determine the angular distribution of the signal generated by the photoemitted electrons. b, Real-size XUV FEL (blue) and IR (red) spatial profiles at waist position. He atoms are also represented as dots with exaggerated dimensions. c, VMI image obtained for high IR intensity (about $3 \times 10^{17} \mathrm{~W} / \mathrm{m}^{2}$ ), right-handed IR polarization and positive topological charge $\left(m_{\mathrm{OAM}}=+1\right)$. d, Same as panel c, with negative topological charge $\left(m_{\mathrm{OAM}}=-1\right)$. 


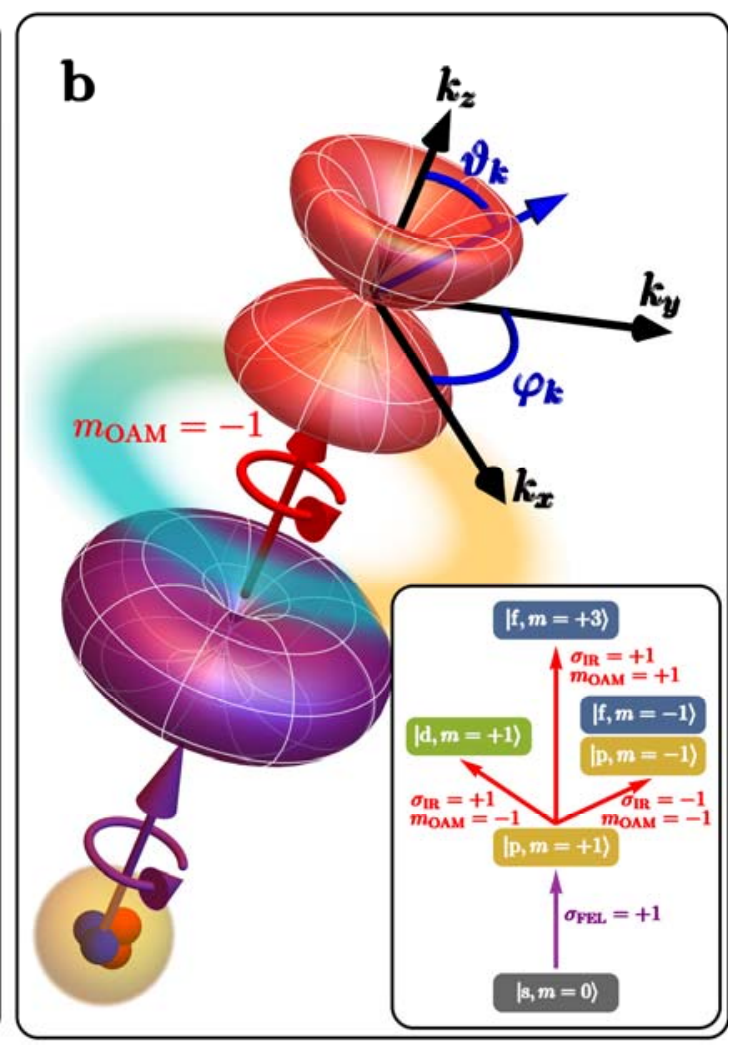

Fig. 2 Theoretical description of the imprinting of light OAM on the matter wave of a single photoelectron released from a He atom. a, Ideal case of a single He atom placed at the centre of the OAM vortex. The photoelectron wave is launched by the VUV radiation (purple arrow), with right-handed circular polarization. This gives rise to a donut-like electron distribution extending in the plane perpendicular to light propagation. The photoemission process is assisted by an IR light vortex (red arrow), carrying right-handed circular polarization $\left(\sigma_{\mathrm{IR}}=+1\right)$ and a positive topological charge $\left(m_{\mathrm{OAM}}=+1\right.$, phase increasing clockwise). The vortex induces a quadrupole continuum-continuum transition, which leads to a donut-like electron distribution extending in the plane perpendicular to light propagation. For the simulations, an IR intensity of $0.7 \times 10^{17} \mathrm{~W} / \mathrm{m}^{2}$ has been used. b, Same as panel a, with $m_{\mathrm{OAM}}=-1$ (phase increasing counterclockwise). In this case, the vortex-spin combination induces a dipole transition, resulting in an electron distribution stretched in the direction of beam propagation. Inset: Selection rules for the different combinations of polarization and topological charge considered in the present work. 

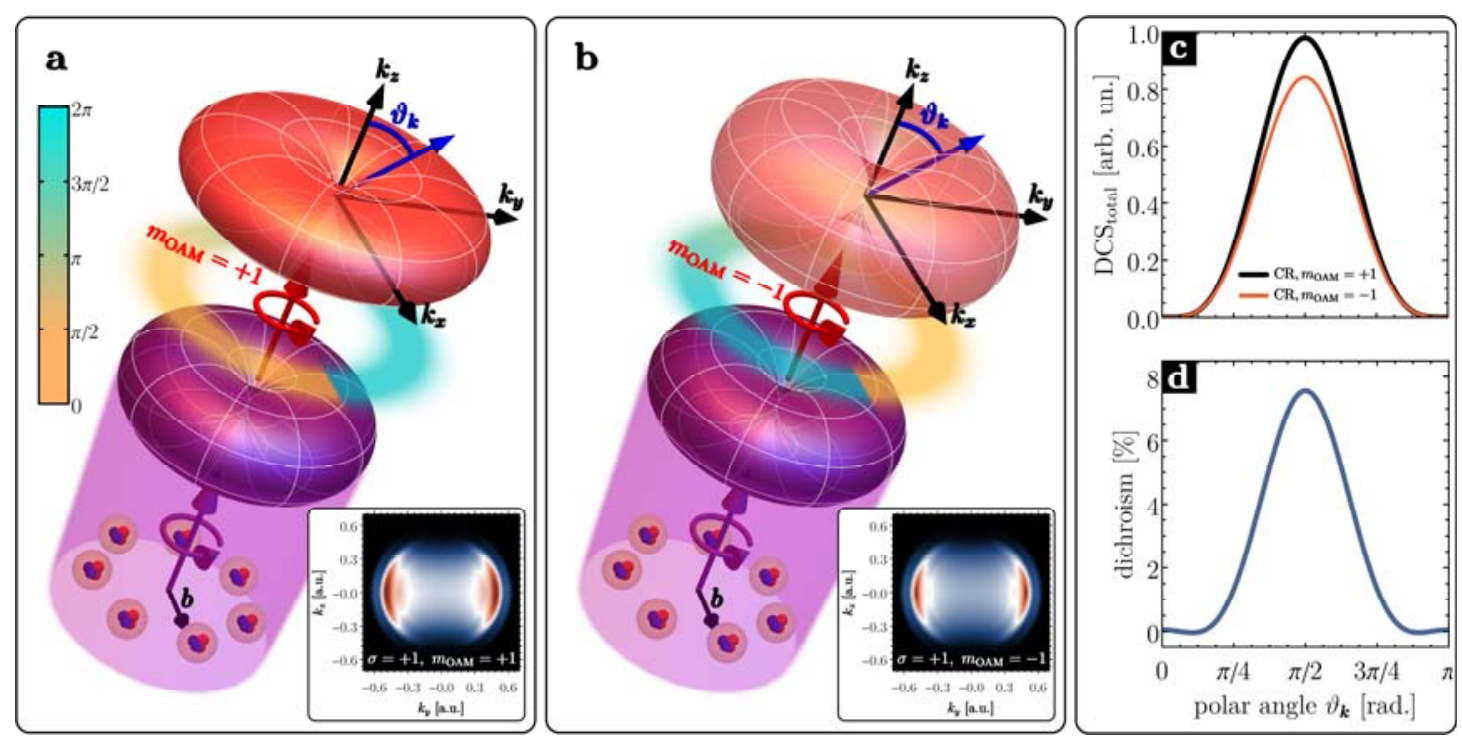

Fig. 3 | Theoretical description of the imprinting of light OAM on the matter waves of photoelectrons released from a sample of $\mathrm{He}$ atoms. a, Sample of $\mathrm{He}$ atoms placed at random positions $\boldsymbol{b}$ from the IR optical axis. The electron distributions generated after sample's interaction with the FEL and IR beams have been found by solving the theoretical problem for different values of $\boldsymbol{b}$ and averaging the obtained results. The parameters are the same as in Fig. 2a; in particular, $\sigma_{\mathrm{IR}}=$ +1 and $m_{\mathrm{OAM}}=+1$. Inset: projection of the photoelectron distribution on the $\left(k_{y}, k_{z}\right)$ plane. b, Same as Fig. a, with $\sigma_{\mathrm{IR}}=+1$ and $m_{\mathrm{OAM}}=-1$. Inset: projection of the photoelectron distribution on the $\left(k_{y}, k_{z}\right)$ plane. c, DCSs obtained by integrating the signal of the first sideband of the projected distributions shown in the insets of panels (a) and (b), as a function of the polar angle. d, OAM-dependent dichroism, defined as the difference between the curves shown in panel (c), normalized to their sum. 

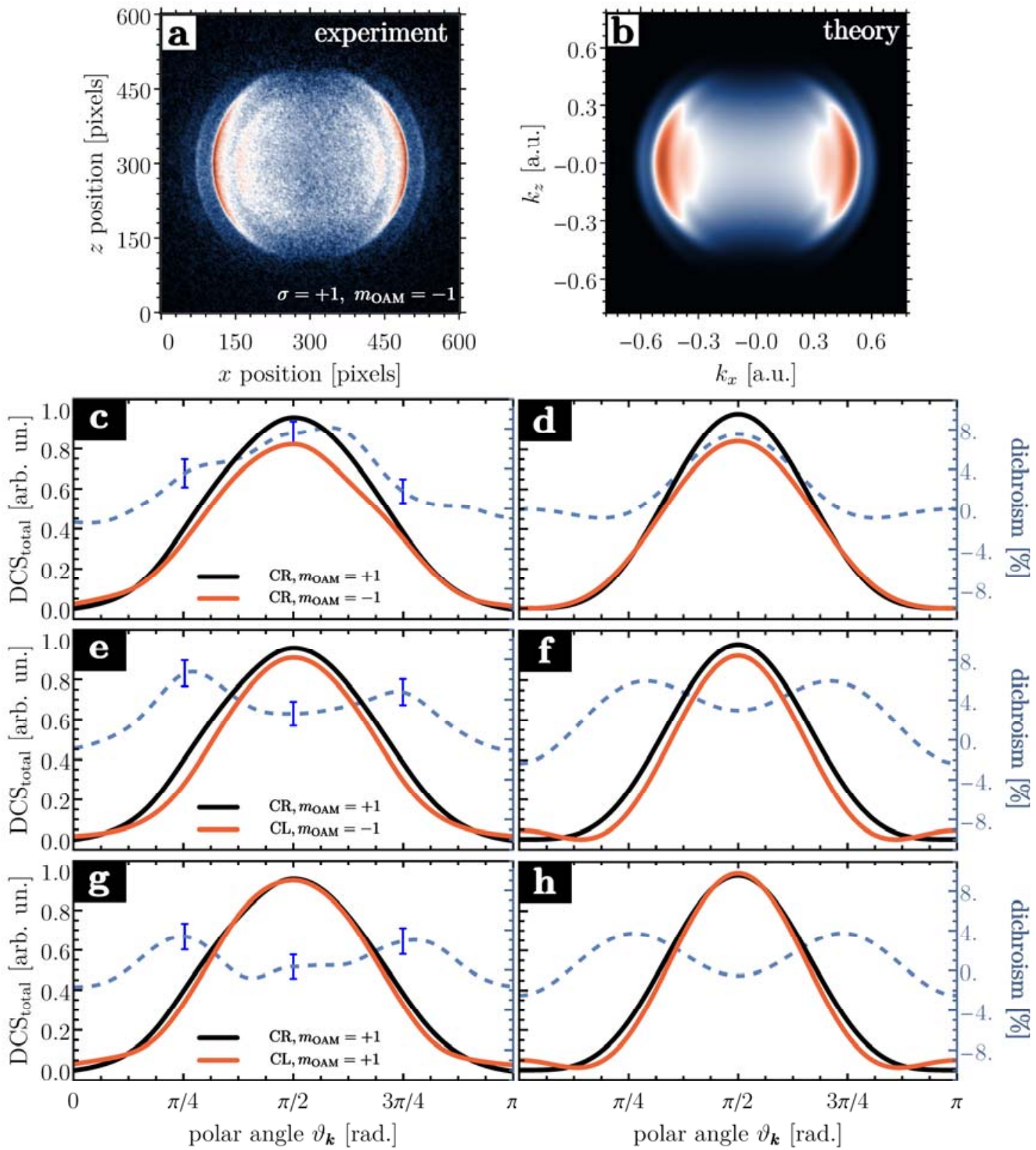

Fig. 4 | Experimental vs. theoretical photoelectron spectra and DCSs for different combinations of IR spin and OAM.

a, Experimental VMI spectrum for the combination $\sigma_{\mathrm{IR}}=+1, m_{\mathrm{OAM}}=-1$. b, Theoretical VMI spectrum for the same combination as in a. c, Continuous curves: experimental DCSs for the combinations $\sigma_{\mathrm{IR}}=+1, m_{\mathrm{OAM}}=+1$ and $\sigma_{\mathrm{IR}}=$ $+1, m_{\mathrm{OAM}}=-1$. Dashed curve: corresponding dichroic contrast. d, Theoretical DCSs and dichroic contrast for the same combination as in c. e, Experimental DCSs and dichroic contrast for the combinations $\sigma_{\mathrm{IR}}=+1, m_{\mathrm{OAM}}=+1$ and $\sigma_{\mathrm{IR}}=$ $-1, m_{\mathrm{OAM}}=-1$. f, Theoretical DCSs and dichroic contrast for the same combination as in e. g, Experimental DCSs and dichroic contrast for the combinations $\sigma_{\mathrm{IR}}=$ $+1, m_{\mathrm{OAM}}=+1$ and $\sigma_{\mathrm{IR}}=-1, m_{\mathrm{OAM}}=+1$. $\mathbf{h}$, Theoretical DCSs and dichroic contrast for the same combination as in $\mathbf{g}$. For a discussion about the evaluation of error bars, see Methods. 


\section{References}

[1] Allen, L. et al. Angular momentum of light and the transformation of LaguerreGaussian laser modes. Phys. Rev. A 45, 8185 (1992).

[2] Kaneyasu, T. et al. Limitations in photoionization of helium by an extreme ultraviolet optical vortex. Phys. Rev. A 95, 023413 (2017).

[3] Schmiegelow, C. T. et al. Transfer of optical orbital angular momentum to a bound electron. Nat. Commun. 7, 12998 (2016).

[4] Afanasev, A. et al. Experimental verification of position-dependent angularmomentumselection rules for absorption of twisted light by a bound electron. New J. Phys. 20023032 (2018).

[5] Allaria, E. et al. Highly Coherent and Stable Pulses from the FERMI Seeded FreeElectron Laser in the Extreme Ultraviolet. Nat. Photonics 6, 699 (2012).

[6] Köksal, K, Berakdar, J. Charge-current generation in atomic systems induced by optical vortices. Phys. Rev. A 86, 063812 (2012).

[7] Picón, A., Mompart, J., Vázquez de Aldana, J.R., Plaja, L., Calvo, G.F., Roso, L. Photoionization with orbital angular momentum beams. Opt. Express 18, 3660 (2010).

[8] Wätzel J., Pavlyukh Y., A Schäffer A.F., Berakdar J. Optical vortex driven charge current loop and optomagnetism in fullerenes Carbon 99, 439 (2016).

[9] Lyamayev, V. A modular end-station for atomic, molecular, and cluster science at the low density matter beamline of FERMI@Elettra. J. Phys. B 46, 164007 (2013). [10] Glover, T. E., Schoenlein, R. W., Chin, A. H. \& Shank, C. V. Observation of laser assisted photoelectric effect and femtosecond high order harmonic radiation. Phys. Rev. Lett. 76, 2468 (1996).

[11] Meyer, M., Costello, J. T., Düsterer, S., Li, W. B. \& Radcliffe, P. Two-colour experiments in the gas phase. J. Phys. B 43, 194006 (2010).

[12] O'Keeffe, P. et al., Polarization effects in two-photon nonresonant ionization of argon with extreme-ultraviolet and infrared femtosecond pulses. Phys. Rev. A 69, 051401 (2004).

[13] Meyer, M. et al. Polarization Control in Two-Color Above-Threshold Ionization of Atomic Helium. Phys. Rev. Lett. 101, 193002 (2008).

[14] Guyétand, O. et al. Evolution of angular distributions in two-colour, few-photon ionization of helium, J. Phys. B: At. Mol. Opt. Phys. 41, 051002 (2008).

[15] Haber, L.H., Doughty, B., Leone, S.R. Photoelectron Angular Distributions and Cross Section Ratios of Two-Color Two-Photon Above Threshold Ionization of Argon. J. Phys. Chem. A 113, 4713152 (2009).

[16] Haber, L.H., Doughty, B., Leone, S.R. Energy-dependent photoelectron angular distributions of two-color two-photon above threshold ionization of atomic helium. Phys. Rev. A 84, 013416 (2011).

[17] O'Keeffe, P. et al. Near-threshold photoelectron angular distributions from twophoton resonant photoionization of He. New J. Phys 15, 013023 (2013).

[18] Grum-Grzhimailo, A.N., Gryzlova, E.V. Nondipole effects in the angular distribution of photoelectrons in two-photon two-color above-threshold atomic ionization. Phys. Rev. A 89 043424-1 (2014).

[19] Taïeb R. et al. Circular dichroism from unpolarized atoms in multiphoton multicolor ionization. Phys. Rev. A 62, 013402 (2000).

[20] Kazansky, A.K., Grigorieva, A.V., Kabachnik, N.M. Circular Dichroism in Laser-Assisted Short-Pulse Photoionization. Phys. Rev. Lett. 107, 253002 (2011). 
[21] Mazza, T. et al., Determining the polarization state of an extreme ultraviolet free480 electron laser beam using atomic circular dichroism. Nat. Commun. 5, 3648 (2014).

481 [22] Quinteiro, G.F., Schmidt-Kaler, F., Schmiegelow, C.T., Twisted-Light-Ion 482 Interaction: The Role of Longitudinal Fields. Phys. Rev. Lett. 119, 253203 (2017).

483 [23] Vrakking, M. J. J. An iterative procedure for the inversion of two-dimensional 484 ion/photoelectron imaging experiments. Rev. Sci. Instrum. 72, 4084 (2001).

485 [24] Zangrando, M. et al. PADReS: the photon analysis delivery and reduction system 486 at the FERMI@Elettra FEL user facility. Rev. Sci. Instrum. 80, 305 (2009).

487 [25] Raimondi, L. et al. Kirkpatrick-Baez active optics system at FERMI: system 488 performance analysis. J. Synchrotron Rad. 26, 1462 (2019).

489 [26] Svetina, C et al. The Low Density Matter (LDM) beamline at FERMI: optical 490 layout and first commissioning, J. Synchrotron Rad. 22, 538 (2015).

491 [27] Sarsa, A., Gálvez, F., Buendia, E. Parameterized optimized effective potential for 492 the ground state of the atoms He through Xe. At. Data Nucl. Data Tables 88, 163 493 (2004). 